\title{
Regge Analysis of Correlations in the Central Plateau*
}

\author{
R. F. Amann and. K. Geer
}

Physics Department, Syracuse University, Syracuse, N. Y. 13210

*Work supported by the U.S. Atomic Energy Commission

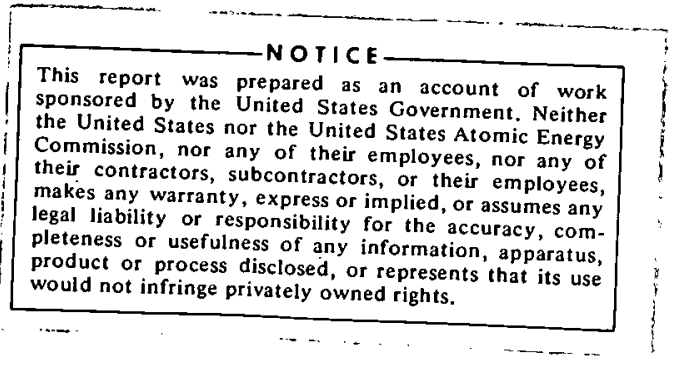




\section{DISCLAIMER}

This report was prepared as an account of work sponsored by an agency of the United States Government. Neither the United States Government nor any agency Thereof, nor any of their employees, makes any warranty, express or implied, or assumes any legal liability or responsibility for the accuracy, completeness, or usefulness of any information, apparatus, product, or process disclosed, or represents that its use would not infringe privately owned rights. Reference herein to any specific commercial product, process, or service by trade name, trademark, manufacturer, or otherwise does not necessarily constitute or imply its endorsement, recommendation, or favoring by the United States Government or any agency thereof. The views and opinions of authors expressed herein do not necessarily state or reflect those of the United States Government or any agency thereof. 


\section{DISCLAIMER}

Portions of this document may be illegible in electronic image products. Images are produced from the best available original document. 
ABSTRACT

1 Recent experimental data on correlations in the central plateau are shown to be consistent with a simple Mueller Rage analysis. The charge dependence of these correlations is in remarkable agreement with both that expected from an extension of the Reggae analysis of single particle production and from charge conservation sum rules. Predictions for the charge dependence of $K-\pi$ correlations are made: 
Recently experiments at $\operatorname{ISR}(1,2)$ and NAL (3) have been conducted to measure correlations between particles produced in the central plateau. Surprisingly, these experiments have confirmed (1) the simple Mueller Regge analysis (4) predictions of short range correlations dependent only upon the rapidity difference $\Delta y$ between two particles in the central region and with a correlation length L of 2., as we would expect for an intercept difference, $\alpha_{P}-\alpha_{R}$, between Pomeron and secondary Reggeon intercepts, of .5. This agreement is surprising in that the form

$$
r_{a b} \exp \{-\Delta y / 2 .\}
$$

describes the correlation between particles a and b not only for large $\Delta y$ (of order $3 ;)_{0}$ but also for small $\Delta y$, and in fact even for $\Delta y=0$. This would be analogous, in terms of the more familiar total cross sections, to a successful description of the total cross section by secondary Regge trajectories over the s or center of mass squared energy range of .2 to $5 .(\mathrm{BeV} / \mathrm{C})^{2}$. Thus this agreement places strong bounds on the contribution of lower Regge trajectories. When combined with the known consistency (5) of the measured approach. to the central plateau. with the $\mathrm{s}^{-\frac{1}{4}}$ prediction of secondary Regge dominance, it suggests that a detailed Regge analysis, with $P, \rho$, and $f$ trajectories, should be attempted. Assuming simple factorizing Regge poles, the Regge analysis prediction for the dimensionless correlation between partiles a and b produced in the central plateau in the reaction $c+d \rightarrow a+b+$ anything is 
$-2-$

$$
\begin{aligned}
& C_{a b}\left(y_{a}, y_{b}\right)=\frac{1}{\sigma_{\infty}^{c d}}\left\{\frac{d^{2} \sigma}{d y_{a} d y_{b}}=\frac{1}{\sigma_{\infty}^{c d}} \quad \frac{d \sigma}{d y_{a}} \cdot \frac{d \sigma}{d y_{b}}\right\}
\end{aligned}
$$

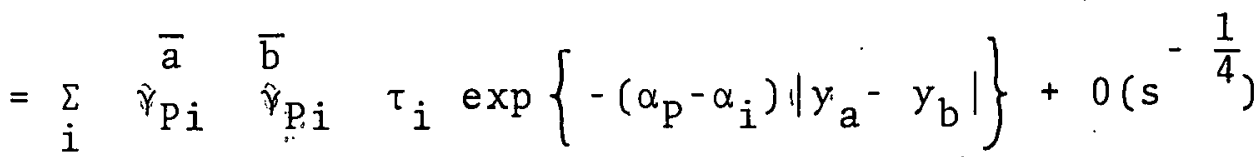

Here $\sigma_{\infty} \cdot c d$ is the asymptotic total cross section; $\dot{\gamma}_{i j}$ is the double Rage coupling for a produced particle b with Reggeons $i$ and $j$ exchanged and $\tau_{i}= \pm 1$ is the signature of the Reggeon $i$. Note that the asymptotic prediction is independent of the incident particles $c$ and $\dot{d}$ and that the approach to this limit is, as with single particle production, expected to be as $s^{-\frac{1}{4}}$.

For either a or $b$ a pion, $G$ parity restricts the sum $i$ to the $\rho$ and $f$ trajectories.'We neglect lower trajectories with similar quantum numbers as is indicated by $C^{\prime} s$ dependence on $\Delta y=\left|y_{a}-y_{b^{\prime}}\right|(1)$. If we define

$$
r_{a b}=\lim _{s \rightarrow \infty} C_{a b}(0,0)
$$

then

$$
\begin{aligned}
& r_{\pi^{+}, \pi^{+}}=r_{\pi^{-}, \pi^{-}}=\left(\gamma_{\mathrm{P} f}^{\left.\pi^{2}\right)^{2}}\right)^{2}\left(\gamma_{\mathrm{P}, \rho}^{\pi}\right)^{2} \\
& r_{\pi^{+}, \pi^{-}}=\left(\gamma_{\mathrm{Pf}}^{\pi}\right)^{2}+\left(\gamma_{\mathrm{P} \rho}\right)^{2} \\
& \frac{1}{2} r_{\mathrm{ch}, \pi^{\circ}}=r_{\pi^{*}, \pi^{\circ}}=r_{\pi^{-}, \pi^{\circ}}=\left(\gamma_{\mathrm{Pf}}{ }^{\pi} \cdot\right)^{2} \\
& r_{\mathrm{ch}, \mathrm{ch}}=4\left(\gamma_{\operatorname{Pff}}^{\pi}\right)^{2}
\end{aligned}
$$


where we have introduced the subscript ch to denote the sum over both $\pi^{+}$'s and $\pi^{-}$'s. Note that the Regge analysis immediately predicts

$$
\mathrm{r}_{\mathrm{ch}, \mathrm{ch}}>0
$$

and

$$
\mathrm{r}_{\pi^{+}, \pi^{-}}>\frac{1}{4} \mathrm{r}_{\mathrm{ch}, \mathrm{ch}}>\mathrm{r}_{\pi^{-}, \pi^{-}}^{-}
$$

These inequalities would hold if possible lower trajectories were included.

The experimental data, with which we wish to compare eqs. (5) through (10), are the quoted values for

$$
\tilde{C}_{a b}\left(\eta_{a}, \eta_{b}\right)=\sigma_{I} \cdot \frac{d^{2} \sigma}{d \cdot n_{a} d \cdot \eta_{b}}\left\{\frac{d \sigma}{d \eta_{a}} \frac{d \sigma}{d \cdot \eta_{b}}\right\}-l^{-1}
$$

and

$$
\tilde{\mathrm{r}}_{\mathrm{ab}}=\tilde{\mathrm{C}}_{\mathrm{ab}}(0,0)
$$

Here $\dot{\sigma}_{I}$ is the inelastic pp cross section and $n_{a}$ is the "1aboratory rapidity" of particle a. This comparison involves the following assumptions:

(a) All produced particles are pions. This is required since these experiments identify only the charges of the produced particles.

(b) Knowledge of $\frac{d \sigma}{d y}$. We have taken the values of these directly from the fits of reference 5. 
(c) The neglect of the non-asymptotic terms in eq. (3). An estimate of the possible error here can be made if we note that the dimensionless single pion production cross section, $\sigma_{\infty}^{-1} \frac{\mathrm{d} \sigma}{\mathrm{dy}}$, is predicted in ref. 5 to rise by $30 \%$ from NAL energies to its asymptotic 1 imit.

(d) Knowledge of the ratio $\sigma_{\infty}^{p p} / \sigma_{I} p p$ which we have set equal to 1.2.

Note that this calculation is independent of any problem with the differences between the true and laboratory rapidities $y$ and $n$. In Table I we list the measured $\tilde{r}_{a b}$ and the calculated $r_{a b}$ for the data of ref. 2 .

The inequalities, eqs.(9) and (10), can be immediately verified from Table.I, as can the approximate validity of the relation

$$
\mathrm{r}_{\mathrm{ch}, \mathrm{ch}}=2 \mathrm{r}_{\mathrm{ch}, \pi^{\circ}}
$$

These values for $r$ imply

$$
\left(\gamma_{\mathrm{P} f}^{\pi}\right)^{2}=.28 \quad\left(\begin{array}{l}
\gamma_{\mathrm{P} \rho} \rho \\
{ }^{2}
\end{array}\right)^{2}=.10
$$

The errors in the above determination can be estimated by noting that if correction (d) above had not been applied the results would be .18 and .08 respectively.

A strong check on the consistency of the above analysis can be made by comparing these values for $\gamma_{P I f}^{\pi}$ and $\gamma_{P}{ }_{0}$, to those needed to describe the $s^{-\frac{1}{4}}$ approach to asymptopia for single pion production : 
in the central region. For $\gamma_{\text {Pf }}^{\pi}$ this check is ambiguous, as the single pion spectra for different incident particles are inconsistent with a unique value; however, eq.14 is in reasonable agreement with the value $\gamma_{\mathrm{Pf}}^{\pi}=-.4$ required for pp scattering. For $\gamma_{\mathrm{Pp}}^{\pi}$, eq. (14) is in good agreement with the value of $\gamma_{\mathrm{P} p}^{\pi}=.31$ used in ref.6. Here, in addition to these spectra;... there is the charge conservation sum rule (6) assuming $\rho$ dominance:

$$
\left(\gamma_{\mathrm{P}_{\rho}}^{\pi}\right)^{2}=\frac{\alpha_{\mathrm{P}}-\alpha_{\rho}}{-4} \gamma_{\mathrm{P} P}^{\pi}
$$

which, for $\gamma_{\mathrm{PP}}^{\pi}=.75$ (5) and $\alpha_{\rho}=.5$, is in excellent agreement with eq. (14) .

The tests of Regge theory and dominance embodied in eqs.(14) and (15) can be alternatively viewed as a measurement of an effective intercept difference $\delta_{\rho}=\alpha_{\mathrm{P}}-\alpha_{\rho}$ averaged over all trajectories with $:$ the cquantumunumbers of the $\rho$. Letting $\lambda$ denote the sum over isovector, even G-parity trajectories, eqs.(5) and (6) generalize to

$$
\therefore \frac{1}{2}\left(r_{\pi^{+}, \pi^{-}}-r_{\pi^{*}, \pi^{-}}\right)=\sum_{\lambda^{\prime}}\left(\gamma_{\mathrm{P}, \lambda}{ }^{\pi}\right)^{2}
$$

and. eq. (15) to

$$
\sum_{\lambda} \frac{\left({ }_{p}^{\bar{\gamma} \lambda}\right)^{2}}{\alpha_{\mathrm{P}}-\alpha_{\lambda}}=\frac{1}{4} \gamma_{\mathrm{PP}}^{\pi}
$$


Then

$$
\delta_{i=}=2\left(\gamma_{P_{P}}^{\pi}\right)^{-1}\left(r_{\pi^{+}, \pi^{-}}-r_{\pi^{-}, \pi^{-}}\right)
$$

defines an experimentally measurable effective intercept difference, which, for the values used above, is .5.3, thus confirming $\rho$ dominance of the sum over $\lambda$. A similar analysis can be performed for the isoscalar, even G parity exchanges. Here, due to the absence of a. sum rule analogous to eq. (17), the integrated charged particle correlation, $f_{2} \mathrm{ch}, \mathrm{ch}$, must be used:

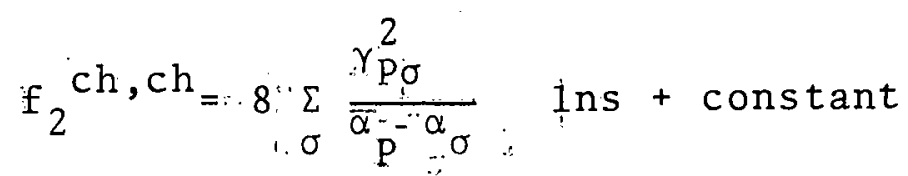

where o runs over all isoscalar, even.G-parity trajectories.

Similar charge conservation sum rules, extended to allow for. strange particle production, (7), imply

$$
\gamma_{\mathrm{P} \rho}^{\mathrm{K}} / \gamma_{\mathrm{P} P}^{\pi}=.5 \quad \gamma_{\mathrm{PP}}^{\mathrm{K}} / \gamma_{\mathrm{PP}}^{\pi}
$$

Accepting the value of .I for the asymptotic $\mathrm{K}^{-} / \pi^{-}$ratio in the central region we then predict

$$
\mathrm{r}_{\mathrm{K}^{-} \pi^{+}}{ }^{-} \mathrm{r}_{\mathrm{K}^{-}}, \pi^{-}=2 \gamma_{\mathrm{P} \rho}^{\pi} \gamma_{\mathrm{P} \rho}^{\mathrm{K}}=.010
$$

Here the smaller size is mostly due to the suppressed K production: We are grateful to Drs. M. Blackmon and T. Ferbel for helpful conversations. 


\section{References}

1.) Pisa- Stony Brook collaboration, as reported by J: Sens at the Washington A 'S meeting (Apri1, 1973).

2.) G. Neuhofer et al. (CERN-Hamburg-Vienna collaboration), as reported at the Vanderbuilt meeting (March, 1973)

3.) C. Moore et al. (Argonne Nat. Lab. - Nat. Accelerator Lab. Stony Brook collaboration), as reported at the Washingtons meeting (April, 1973).

4.) A. H. Mueller, Phys: Rev. D2 (1970) 2963.

5.) T. Ferbel, Phys. Rev. Letters 14 (1972) 448.

6.) R. F. Amann and Maurice L. Blackmon, to be published in Phys. Letters. B.

7.) R. F. Amann, Maurice L. Blackmon, and. K. Geer, to be published. 


\section{TABLE I}

Reported values for $\tilde{r}_{a b}$ compared to the calculated $r_{a b} \because \ldots .$.

\begin{tabular}{cccc} 
ref. & $\mathrm{ab}$ & $\tilde{\mathrm{r}}_{\mathrm{ab}}$ & $\mathrm{r}_{\mathrm{ab}}$ \\
\hline 3 &,+- & .80 & .35 \\
3 & $\mathrm{ch,ch}$ & .60 & 1.11 \\
3 &,-- & .35 & .15 \\
2 & $\mathrm{ch, \pi ^{ \circ }}$ & .62 & .57 \\
\hline
\end{tabular}

